\title{
NFATc1 regulates cell proliferation, migration, and invasion of ovarian cancer SKOV3 cells in vitro and in vivo
}

\author{
LONG LI $^{1}$, ZHAONING DUAN ${ }^{2}$, JIHUI YU ${ }^{1}$ and HONG-XING DANG ${ }^{3}$ \\ Departments of ${ }^{1}$ Physical Examination and ${ }^{2}$ Gynecology, The First Affiliated Hospital of Chongqing Medical University; \\ ${ }^{3}$ Department of PICU, Children's Hospital of Chongqing Medical University, Ministry of Education Key Laboratory of \\ Child Development and Disorders; China International Science and Technology Cooperation Base of Child Development \\ and Critical Disorders; Chongqing Engineering Research Center of Stem Cell Therapy, Chongqing 400016, P.R. China
}

Received January 14, 2016; Accepted February 20, 2016

DOI: $10.3892 /$ or.2016.4904

\begin{abstract}
NFATc1 (nuclear factor of activated T-cells c1) is associated with malignancy in several cancer models. However, the expression and function of NFATc1 in ovarian cancer remain elusive. In the present study, we investigated the role of NFATc1 in human epithelial ovarian cancer (EOC) using human ovarian adenocarcinoma SKOV3 cells and patient characteristics. NFATc1 expression was silenced by siRNA in the SKOV3 ovarian cancer cell line and in human ovarian cancer nude mouse xenografts. Real-time PCR, western blotting, immunohistochemical staining, MTT, flow cytometry, Transwell, erasion trace and mouse assays were used to detect NFATc1 expression, cell proliferation, apoptosis, cell invasion and migration, tumor growth and angiogenesis. Survival analysis was performed to assess the correlation between NFATc1 expression and survival. NFATc1 was overexpressed in the SKOV3 ovarian cancer cell line and in human serous/mucinous ovarian cancer tissues. The silencing of NFATc1 expression by siRNA reduced cell proliferation and migration and promoted apoptosis in vitro and decreased the ovarian cancer cell tumorigenesis in vivo in nude mice. NFATc1 overexpression in high-grade serous ovarian carcinomas was an independent prognostic factor of poor overall survival and of early relapse $(\mathrm{P}<0.01)$ in a univariate analysis. Our present data provide evidence that NFATc1 is overexpressed in human serous/mucinous ovarian cancer and is associated with a poor prognosis. NFATc1 silencing regulates the cell cycle, apoptosis, invasion and migration. NFATc1 thus has the potential to be a therapeutic target and to be used in EOC diagnosis and prognosis.
\end{abstract}

Correspondence to: Dr Hong-Xing Dang, Department of PICU, Children's Hospital of Chongqing Medical University, Chongqing 400016, P.R. China

E-mail: docdang@163.com

Key words: NFATc1, cytoplasmic 1, ovarian cancer, invasion, poor prognosis

\section{Introduction}

The nuclear factor of activated T-cell (NFAT) family is a multigene family of $\mathrm{Ca}^{2+} /$ calcineurin-dependent transcription factors that were initially identified in T-lymphocytes (1) and is composed of four classical calcium-responsive members, NFATc1, NFATc2, NFATc3 and NFATc4, and the new recently cloned member calcium insensitive NFAT5 (2). Each of these proteins appears to be expressed as several isoforms by alternative splicing. NFAT proteins are confined to the cytoplasm of unstimulated cells in a highly phosphorylated form under basal conditions. After an increase in intracellular calcium, NFAT proteins are dephosphorylated by the serine/threonine phosphatase calcineurin and translocate to the nucleus. Once in the nucleus, they bind to consensus DNA sequences, activating the transcription of NFAT-dependent genes (3), and its expression is associated with other essential transcription factors, such as AP-1 (4), GATA-4, EGR, MEF2 and the important oncogene FOXP (5). In addition to the immune system, NFAT is functionally important in osteoclast differentiation (6), endothelial cell differentiation in cardiac valve morphogenesis (7) and in preventing organ transplant rejection (8).

Over the past 20 years, evidence supports a role for NFAT signaling in cell growth and development, and it is expressed not only in lymphocytes but also in other histocytes (including epithelial cells) (9-11). Some researchers have further found that NFAT is also closely related to tumorigenicity (12). The subtypes of the NFAT family have unique functions; among them, NFATc1 (nuclear factor of activated T-cells c1) (also referred to as NFAT2) has been demonstrated to be associated with tumorigenesis (13-15). Studies have found that NFATc1 plays an essential role in the regulation of fibroblast NIH 3T3 cells through the induction of the tumoral phenotype, which indicates that NFATc1 is a potential oncogene (16). In addition, as previously mentioned, NFATc1 proteins are dephosphorylated by calcineurin, and calcineurin plays an essential role in the development of malignant lymphomas (17). Recent evidence also has confirmed that NFATc1 is overexpressed in other malignancies and is a key factor in their development, such as prostate (18) and pancreatic cancers (19).

NFATc1 is overexpressed in human solid tumors and hematological malignancies (20) and is also functionally important in 
tumor cell autonomic function and is involved in diverse functions, including tumor cell differentiation, invasion and escape from the immune system. Furthermore, it has been shown to play a key role in tumor angiogenesis (21). Embryogenesis studies have shown that NFATc1 has a vital role in cardiovascular development (22), and it modulates endothelium growth, differentiation and cell cycle progression (23). Dephosphorylated NFATc1 translocates into the nucleus to regulate transcription. Cell activation by a variety of factors, including vascular endothelial growth factor-A (VEGF-A), boosts intracellular calcium, activates calcineurin and induces NFATc1 transcriptional activity $(24,25)$. CXCR2, also known as IL-8RB, was initially recognized as an $\mathrm{ELR}^{+}$transmembrane chemokine receptor expressed on neutrophils. It has a high affinity for $\mathrm{ELR}^{+}$ chemokines, such as IL-8 and Gro-1, and plays a critical role in Gro-1- or IL-8-mediated tumorigenesis, angiogenesis and aggressiveness (26). The present study also studied CXCR2 to observe the role of NFATc1 in EOC invasion and metastasis.

Although little information is available on NFATc1 expression and function in tumor cells, these observations prompted us to examine the potential role of the NFATc1 signaling pathway in EOC. In the present study, we investigated the functional role of NFATc1 in ovarian cancer cells in terms of expression, cell apoptosis and proliferation, and aggressiveness, and we studied COX2 to observe the role of NFATc1 in EOC angiogenesis. We also analyzed the association of NFATc1 expression in high-grade serous ovarian carcinomas with clinical pathologic characteristics, overall disease survival and disease-free survival of patients.

\section{Materials and methods}

Cell lines and NFATc1 siRNA. The SKOV3 ovarian cancer cell line was from the Molecular Medicine and Cancer Center, Chongqing Medical University, and the cells were maintained in medium containing $10 \%$ fetal bovine serum, $2 \mathrm{mmol} / \mathrm{l}$ l-glutamine, penicillin (100 units/ml) and streptomycin (100 $\mu \mathrm{g} / \mathrm{ml})$. A 375-bp NFATc1 primer (5'-TCTGGGAGATG GAAGCAAAGACTG-3'; 5'-AGGGCTATCACGTGGTG TGAAGAG-3') and a 180-bp primer (5'-GCCCTGACAGC TCCCAAGCCT-3'; 5'-ATGCGTCATGCCGCTTCCCAG-3') were designed for PCR. Three DNA oligos (siRNA-880, antisense, 5'-UUCCGGCACAGUCAAUGACGGCUCG-3' and sense, 5'-CGAGCCGUCAUUGACUGUGCCGGAA-3'; siRNA-1169, antisense, 5'-AGAGAAUUCGGCUUGCACA GGUCCC-3' and sense 5'-GGGACCUGUGCAAGCCGAA UUCUCU-3'; and siRNA-1307, antisense 5'-AGACGUAGA AACUGACGUGAACGGG-3' and sense 5'-CCCGUUCACG UCAGUUUCUACGUCU-3') were designed as green fluorescent small interfering RNA (siRNA) against NFATc1 mRNA to target the open reading frame of NFATc1 cDNA. These DNA oligos were contained in Lipofectamine ${ }^{\mathrm{TM}} 2000$ and were used to infect SKOV3 cells; the highest transfection efficiency oligo was selected by RT-PCR to use for interference. The subjects can be divided into three groups as follows according to the intervention measures: A, Blank; B, Control and C, siRNA.

\section{Patient tissue specimens}

Samples. A total of 156 patient samples were collected from the Department of Pathology at the First Affiliated Hospital of Chongqing Medical University, and the sample group was comprised of the following: 96 EOCs (malignant group), 30 serous (mucinous) cystadenomas (control group) and 30 benign ovarian endometriotic cysts (control group). All slides were reviewed twice by two pathologists to verify the diagnoses.

The use of tissue blocks and the chart review were approved by the Institutional Review Board of the Chongqing Medical University. The archived tissue blocks were retrieved from the Department of Pathology at Chongqing Medical University. The selection of patient tissues was not based on treatment. Follow-up information was updated from 2010 to 2014 by reviewing medical records at the First Affiliated Hospital of Chongqing Medical University. The randomly selected formalin-fixed paraffin-embedded tissues included high-grade serous and mucinous ovarian carcinomas $(n=96)$, and the serous (mucinous) ovarian cystadenoma $(n=30)$ and ovarian endometriotic cysts $(n=30)$ were matched to the carcinoma samples. Briefly, ovarian tissue blocks were selected by reviewing hematoxylin and eosin-stained sections by two pathologists and constructed by taking core samples from morphologically representative areas of paraffin-embedded tumor tissues and assembling them on a recipient paraffin block. For each case, two replicate samples with 1-mm core diameter were collected, and each was placed on a separate recipient block. All samples were spaced $0.5 \mathrm{~mm}$ apart. Five-micrometer sections were obtained from the microarray and stained with hematoxylin and eosin to confirm the presence of tumor and to assess tumor histology. Sample tracking was based on coordinate positions for each tissue spot in the block; the spots were transferred onto TMA slides for staining.

Tumor formation in nude mice. To evaluate the ability of cells to form tumors, 4- to 8-week-old BALB/c athymic female nude mice from the Animal Experimental Center, Chongqing Medical University were given bilateral injections of SKOV3 tumor cells, with a total of 18 mice used. All mouse experiments were performed in accordance with the institutional guidelines approved by the Institutional Animal Care and Use Committee. Each subcutaneous injection consisted of $5 \times 10^{6}$ cells $(0.2 \mathrm{ml})$ for SKOV3. Control mice were injected with SKOV3 cell lines that expressed NFATc1 siRNA. The mice were kept in a specific pathogen-free environment and were checked every 2 days for 30 days. After 30 days, the mice were sacrificed by exposure to $5 \%$ carbon monoxide. The tumor inhibition rate was calculated with the use of the tumor weight: Tumor inhibition rate $(\%)=($ control weight - experimental weight)/control weight $x 100 \%$. The tumor volume was calculated with the use of the following formula: Tumor volume $\left(\right.$ in $\left.\mathrm{mm}^{3}\right)=\left(\mathrm{Lx} \mathrm{W}^{2}\right) / 2$, where $\mathrm{L}$ is the length and $\mathrm{W}$ is the weight). All tumors for each group were excised, fixed in $10 \%$ formalin overnight, and subjected to routine histologic examination and immunostaining of NFATc1 (antibody cat. no. ab25916, Abcam, 1:200), CK (antibody cat. no. BM0030, BOSTER, 1:500), CD34 (antibody cat. no. SC-9095, Santa Cruz, 1:500) and CXCR2 (antibody cat. no. bs-1629R, Bioss, 1:800) by investigators who were blinded to the tumor status. The assay was repeated twice.

Immunohistochemical staining and analysis. Immunohistochemical staining for NFATc1 (ab25916, 1:200) was performed 
using avidin-biotin-peroxidase methods. Briefly, tissue slides were deparaffinized in xylene and rehydrated in a graded series of ethanol, and the sections were subjected to antigen retrieval by boiling in $0.01 \mathrm{~mol} / 1$ sodium citrate buffer $(\mathrm{pH}$ 6.0) in a microwave oven for $10 \mathrm{~min}$. After blocking endogenous peroxidase activity with $0.3 \%$ hydrogen peroxide and blocking non-specific protein binding with $1.5 \%$ normal goat serum, the sections were incubated overnight with an antibody at $4{ }^{\circ} \mathrm{C}$ in a humidified chamber. Then, the sections were incubated with biotinylated goat anti-mouse IgG for $30 \mathrm{~min}$ and detected with the LSAB system (Dako). Sections were lightly counterstained with hematoxylin. The primary antibody was replaced with $1 \mathrm{X}$ PBS as a negative control. Differences in proportions between NFATc1 expression and FIGO stage, age at diagnosis, family history, relapse, level of debulking surgery, clinical response, the presence of ascites, and chemoresponse were calculated by $\chi^{2}$ analysis or Pearson's correlation as appropriate. Entire tissue sections were evaluated by $\mathrm{H}$-score values, which are objective measurements of staining intensity, and the percentage of tumor cells that stained positive. Five fields of each slice were randomly selected with a magnification of $x 400$, and the numbers of positive cells were counted per 100 cells per field. Staining intensity was scored as negative $(<5 \%$ of positive tumor cells), $1+$ (mild intensity), $2+$ (moderate intensity), or $3+$ (intensity greater than that of the positive control). The sections were evaluated by two pathologists using a double-blind method to determine the immunohistochemistry results. The formula was $\mathrm{H}$-score $=\Sigma(\mathrm{i}+1) \times \mathrm{p}_{\mathrm{i}}$, where $\mathrm{i}$ indicates the intensity of staining, $p_{i}$ was the percentage of cells with positive staining/the total number of tested cells, and 1 was the correction factor.

\section{Real-time PCR}

RNA isolation and complementary DNA synthesis. RNA was isolated from SKOV3 cells (treated by siRNA and control) using RNeasy Mini kits (Qiagen, Santa Clarita, CA, USA) according to the manufacturer's instructions. The RNA was eluted with water, stored at $-70^{\circ} \mathrm{C}$ and evaluated by agarose electrophoresis. For complementary DNA (cDNA) synthesis, $\sim 1 \mu \mathrm{g}$ of total RNA was transcribed with cDNA transcription reagents (PE Applied Biosystems, Foster City, CA, USA) using random hexamers, according to the following conditions: $30^{\circ} \mathrm{C}$ for $10 \mathrm{~min} ; 50^{\circ} \mathrm{C}$ for $20 \mathrm{~min} ; 99^{\circ} \mathrm{C}$ for $5 \mathrm{~min}$; and $5^{\circ} \mathrm{C}$ for $5 \mathrm{~min}$.

The PCR conditions were $94^{\circ} \mathrm{C}$ for $2 \mathrm{~min}$ ( 1 cycle) and $94^{\circ} \mathrm{C}$ for $30 \mathrm{sec}$ and $58^{\circ} \mathrm{C}$ for $30 \mathrm{sec}(30$ cycles).

Gene expression was measured in real-time with the GeneAmp 5700 Sequence detection system (PE Applied Biosystems). The primers and TaqMan probes (Custom Oligo Synthesis Service, Foster City, CA, USA) were designed to span intronic junctions to avoid amplification of genomic DNA and to generate amplicons of fewer than $150 \mathrm{bp}$ to enhance the efficiency of PCR amplification. The probes were labeled at the 5' end with the reporter dye molecule FAM (6-carboxy-fluorescein; emission $\lambda \max =518 \mathrm{~nm}$ ) and at the 3 ' end with the quencher dye molecule TAMRA (6-carboxytetramethyl-rhodamine; emission $\lambda_{\max }=582 \mathrm{~nm}$ ). On amplification, sequence-specific probes that had annealed to the template were cleaved by the 5 nuclease activity of the $T a q$ polymerase reaction. Real-time monitoring of fluorescent emission from the cleavage of probes allowed for defining the cycle threshold during the exponential phase of the amplification. DNA standards were generated by PCR amplification of the gene product and quantification by spectrophotometry. The number of copies was calculated based on the molecular weight of the gene amplicon. Real-time PCRs of cDNA samples and DNA standards were performed in a total volume of $25 \mu \mathrm{l}$ with 1X TaqMan Master Mix (PE Applied Biosystems) and primers (375 bp). The primer sequences were 5'-TCTGGGAGATG GAAGCAAAGACTG-3' and 5'-AGGGCTATCACGTGG TGTGAAGAG-3'. Standard curves were generated for the gene of interest. The solution curve detection conditions were $95^{\circ} \mathrm{C}$ for $1 \mathrm{~min}$ and $70^{\circ} \mathrm{C}$ for $1 \mathrm{~min}$; then, the temperature was increased to $92^{\circ} \mathrm{C}$ by $0.1^{\circ} \mathrm{C}$ per $\mathrm{sec}$, and the fluorescence was detected continuously. The expression level of target gene NFATc1 was $\mathrm{X}=2^{-\mathrm{\Delta CT}}$ approximately when the amplification efficiency was close to 1.0. The $\Delta \mathrm{CT}$ indicates the difference between the target gene NFATc1 and the housekeeping gene.

Western blotting. Total protein extracts from the SKOV3 cells and transplanted tumor tissue were obtained using lysis buffer, and equal amounts (30 $\mu \mathrm{g}$ per load) were analyzed by immunoblotting. An antibody against $\beta$-actin was obtained from Sigma-Aldrich (A5441, 1:20,000). Antibodies used were against NFATc1 (ab25916; 1:1,000), and against CXCR2 (antibody cat. no. bs-1629R, 1:800). The secondary antibodies were anti-rabbit immunoglobulin horseradish peroxidase-linked $\mathrm{F}(\mathrm{ab}) 2$ fragment from donkey (Amersham Biosciences). Western blot reagents were from an electrochemiluminescence kit (Amersham Biosciences).

Cell proliferation and apoptosis. To detect the inhibition rate of cell proliferation, each group of cells in the logarithmic growth phase was plated at $5 \times 10^{3}$ cells/well, and each condition was repeated thrice. The experimental cells were transfected by NFATc1 siRNA. After incubation for 6 to $24 \mathrm{~h}$, $200 \mu \mathrm{l}$ of RPMI-1640 was added per well. The 1640 reagent was removed after $24 \mathrm{~h}$, and after $48 \mathrm{~h}$, RPMI-1640 and $20 \mu \mathrm{l}$ of MTT was added. The cells were cultured for $4 \mathrm{~h}$ at $37^{\circ} \mathrm{C}$; the reagent was then removed, and $150 \mu \mathrm{l}$ of dimethylsulfoxide was added per well. The samples were shaken slowly for $10 \mathrm{~min}$, and the absorbance in each well, including the blanks, was measured at $490 \mathrm{~nm}$ using a microtiter plate reader. The cell growth inhibition rate $(\%)=\left(1-\right.$ experimental group $\mathrm{A}_{490}$ mean value/control group $\mathrm{A}_{490}$ mean value) x $100 \%$.

The number of apoptotic cells was detected by flow cytometry and was analyzed by CellQuest software. To detect apoptosis, $1 \times 10^{5}$ cells were stained with Annexin $\mathrm{V}$ and propidium iodide, according to the Annexin V-Fluorescence Apoptosis Detection Kit I (BD Biosciences) and were subjected to analysis with a FACStation equipped with CellQuest software. The percentage of apoptotic cells was calculated in terms of peaks (M2) in the histogram, representing the early apoptotic population (Annexin $\mathrm{V}^{+}$/propidium iodide ${ }^{-}$). The experiment was performed in duplicate and repeated three times.

\section{Transwell and the erasion trace}

Transwell in vitro migration assays. A total of $70 \mu 1$ of Matrigel $(1 \mathrm{mg} / \mathrm{ml}$ ) was added to the filter (filter pores, $8 \mu \mathrm{m}$ ) for $60 \mathrm{~min}$ to allow for the restructuring of the basilar membrane. SKOV3 cells $\left(1 \times 10^{5} / \mathrm{ml}\right.$ cells in $200 \mu \mathrm{l}$ of DMEM) were added to the 
upper chamber, and 10\% FBS/DMEM (50 $\mu \mathrm{l})$ was added to the lower chamber; the samples were cultured with $5 \% \mathrm{CO}_{2}$ for $24 \mathrm{~h}$ at $37^{\circ} \mathrm{C}$. The cells were removed from the surface of the filter, fixed by methyl alcohol and stained with crystal violet for $15 \mathrm{~min}$, and the cells that migrated through the filter pores and were on the underside of the filter were counted. The number of cells that migrated across the filters were counted in 5 fields per insert, and the values were averaged. For each migration condition, three identical replicates were performed.

In vitro wound-healing assay. SKOV3 cells were treated with siRNA as described above. After incubation for $24 \mathrm{~h}$, the cells were removed by trypsinization, counted and plated at $5 \times 10^{5} / \mathrm{ml}$ in 6 -well dishes. The cells were incubated overnight, yielding confluent monolayers for wounding. Wounds were created using a pipette tip, and images were captured immediately (time zero) and at 24 and $48 \mathrm{~h}$ after wounding. The cell monolayer that migrated from the wounded edge during this time period was counted. The number of migrated cells after siRNA treatment (control and targeted) was compared. Experiments were performed in triplicate and repeated at least five times.

Statistical analyses. The number of mice (sample size) required to reach significance was determined in preliminary pilot studies that used the following formula: $n=16 \times(\mathrm{SD} /$ difference in mean tumor volume $)^{2}+1$. The results of that pilot study indicated that six mice would be required to detect differences in tumor size with $80 \%$ power at a P-value of $<0.05$. Each mouse received two bilateral flank injections, from which, the mean volume of tumor in each mouse generated from $5 \times 10^{6}$ cells (for SKOV3) was computed to determine the growth curve (the mean tumor volume in each group $=$ total mean volume from each mouse divided by the number of mice). Statistical analysis was performed using Fisher's exact test at different time points for the mean tumor sizes of each group. Differences in proportions were evaluated by the Fisher's exact test as appropriate. The correlation between NFATc1 expression in tissue arrays (based on the scores of CXCR2 immunostaining intensity) and patient survival was analyzed by the Kaplan-Meier method using SPSS 17.0 software (SPSS, Inc., Chicago, IL, USA). The clinical correlation in terms of NFATc1 expression and patient survival was evaluated by excluding missing data. The association between the expression of NFATc1 and clinical pathological parameters was analyzed with contingency tables and Pearson W2 test. $\mathrm{P}<0.05$ was considered significant. All statistical tests were two-sided.

\section{Results}

Association of NFATcl with ovarian cancer. We first examined NFATc1 expression in the ovarian cancer tissues by immunohistochemical staining. As shown in Fig. 1A and Table I, epithelial ovarian cancer samples expressed high levels of NFATc1 compared with two types of benign ovarian tumors. Then, using Lipofectamine ${ }^{\mathrm{TM}} 2000$ infection, we delivered siRNA against NFATc1 into the ovarian cancer cell line SKOV3. As shown by immunofluorescence and RT-PCR (Fig. 1B and C; Tables II and III), NFATc1 was notably silenced in cells treated with the specific NFATc1 siRNA compared with the control group, which led to a marked decrease in the
Table I. Immunohistochemical staining and H-score.

\begin{tabular}{lcr}
\hline & Staining rate $(\%)$ & H-score \\
\hline EOC & $93 / 96(96.87)$ & $269(0-356)$ \\
Mucinous (serous) cystadenoma & $6 / 30(20)$ & $75(0-180)$ \\
Ovarian endometriosis cyst & $5 / 30(17)$ & $85(0-185)$ \\
\hline
\end{tabular}

$\mathrm{H}$-score $=\sum(\mathrm{i}+1) \times \mathrm{p}_{\mathrm{i}}$, where $\mathrm{i}$ is the intensity of staining, $\mathrm{p}_{\mathrm{i}}$ is the percentage of cells with positive staining/the total number of tested cells, and 1 is the correction factor, $\mathrm{P}<0.05$ was considered significant. Epithelial ovarian cancer compared to ovarian benign tumor, $\mathrm{P}<0.05$. Epithelial ovarian cancer to ovarian endometriosis cyst, $\mathrm{P}<0.05$. Ovarian benign tumor to ovarian endometriosis cyst, $\mathrm{P}>0.05$.

Table II. Transfection rate of NFATc1 siRNA in each group (\%).

\begin{tabular}{lcc}
\hline Group & $24 \mathrm{~h}(\%)$ & $48 \mathrm{~h}(\%)$ \\
\hline siRNA1 & 54.2 & 59.1 \\
siRNA2 & 72.6 & 85.3 \\
siRNA3 & 50.2 & 51.9 \\
\hline
\end{tabular}

Transfection group at 24 or $48 \mathrm{~h}$ compared with the other groups, $\mathrm{P}<0.01$.

Table III. Statistical analysis of NFATc1 mRNA in each transfection group after treatment with siRNA for $48 \mathrm{~h}$.

\begin{tabular}{lcc}
\hline Group & OD & P-value \\
\hline siRNA1 & $0.532 \pm 0.001$ & P1 \\
siRNA2 & $0.278 \pm 0.001$ & P2 \\
siRNA3 & $0.498 \pm 0.003$ & P3 \\
\hline
\end{tabular}

Transfection group 2 at $48 \mathrm{~h}$ compared with the other groups, $\mathrm{P}<0.01$.

Table IV. Statistical analysis of NFATc1 expression in the transplanted tumors.

\begin{tabular}{lcc}
\hline Groups & NFATc1 & P-value \\
\hline A (Blank) & $0.912 \pm 0.001$ & P1 \\
B (Control) & $0.885 \pm 0.005$ & P2 \\
C (siRNA) & $0.362 \pm 0.003$ & P3 \\
\hline
\end{tabular}

Comparison of each group for NFATc1 expression. P1, A:B >0.05; $\mathrm{P} 2, \mathrm{~B}: \mathrm{C}<0.05 ; \mathrm{P} 3, \mathrm{~A}: \mathrm{C}<0.05$.

secreted expression of NFATc1 as detected by western blotting (Fig. 1D). To investigate whether NFATc1 downregulation is associated with in vitro and in vivo tumor growth in ovarian cancer cells, we investigated cell proliferation and mouse xenograft tumor growth after inoculation. As indicated in Fig. 2 and Table IV, NFATc1 expression (Fig. 2A) and the 

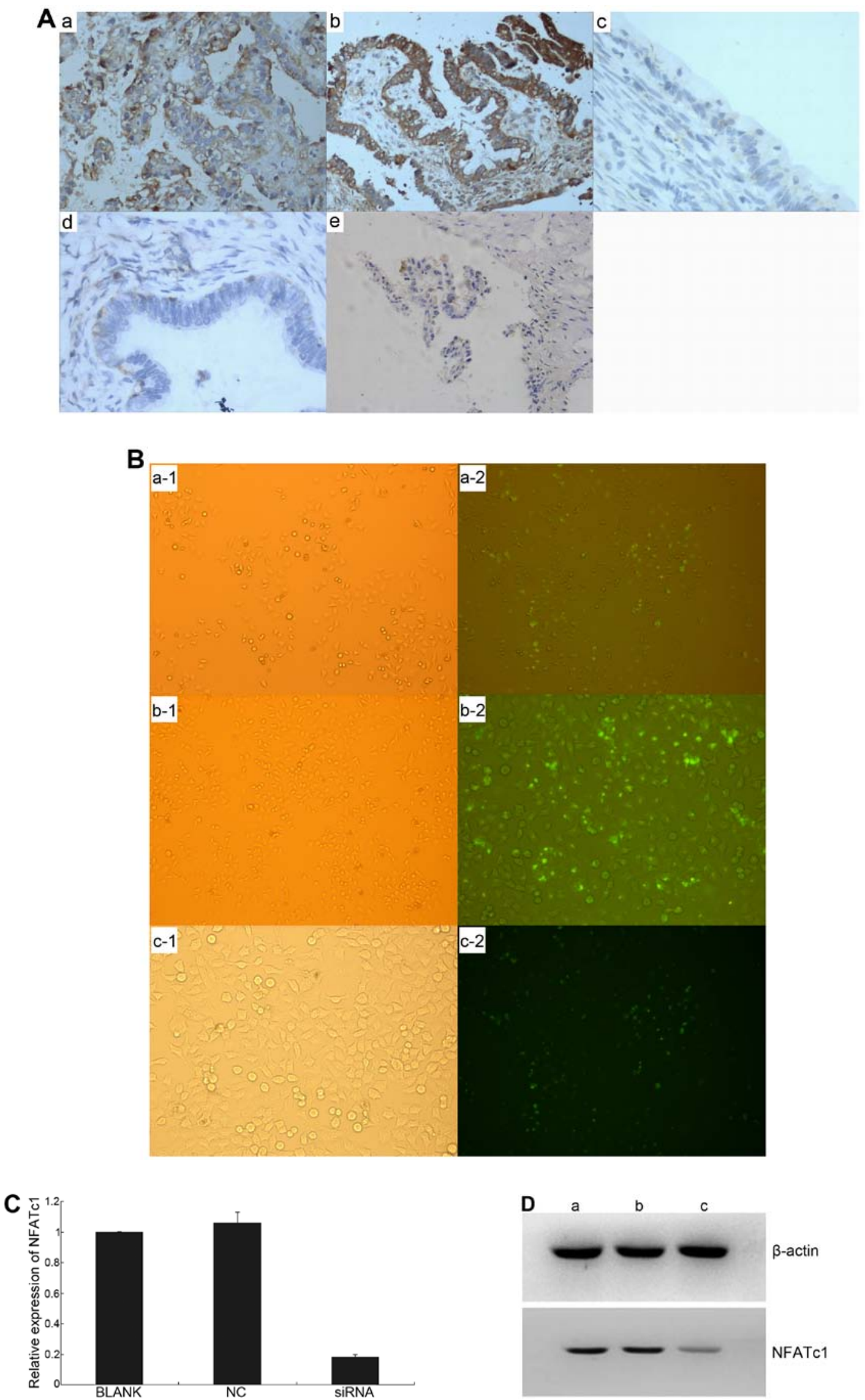

Figure 1. NFATc1 expression was first examined in ovarian cancer tissues by immunohistochemical staining. (A) epithelial ovarian cancer samples expressed high levels of NFATc1 compared with two types of benign ovarian tumors $(\mathrm{P}<0.05)$ (a and b, EOCs; c, serous cystadenoma; d, mucinous cystadenoma; $\mathrm{e}$, ovarian endometriotic cysts) (also Table I). NFATc1 siRNA was transfected into SKOV3 cells, which were then subjected to (B) immunofluorescence tests and (C) real-time reverse transcription polymerase chain reaction (RT-PCR) assays to determine the transfection-induced inhibition rate (a-c, siRNA1-3) (D) NFATc1 was notably silenced in cells treated with the specific NFATc1 siRNA compared with the control group, which led to a marked decrease in the secreted expression of NFATc1 as detected by western blotting. Lane a, control; lane b, blank; and lane c, siRNA). 


\section{B}

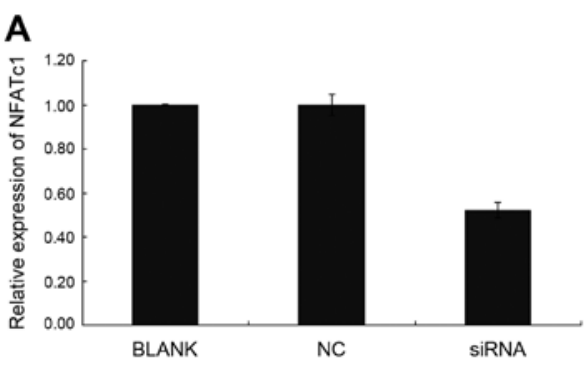

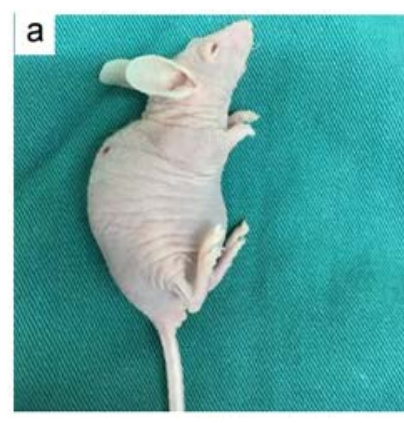

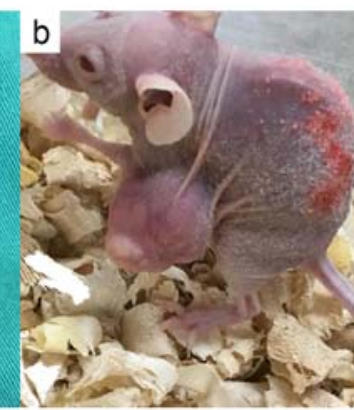

c

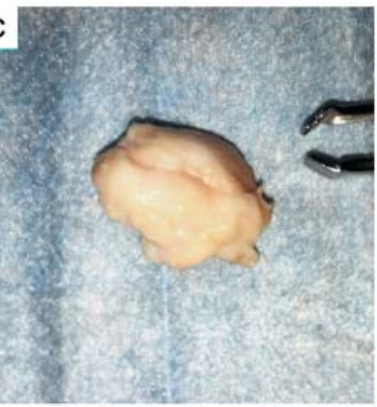

Figure 2. To investigate whether NFATc1 downregulation is associated with in vitro and in vivo tumor growth in ovarian cancer cells, we investigated cell proliferation and mouse xenograft tumor growth after inoculation. (A) NFATc1 expression (also Table IV) and (B) the subcutaneous tumor size in animals (also Table V) were both distinctly reduced in cells treated with NFATc1 siRNA compared with the controls.
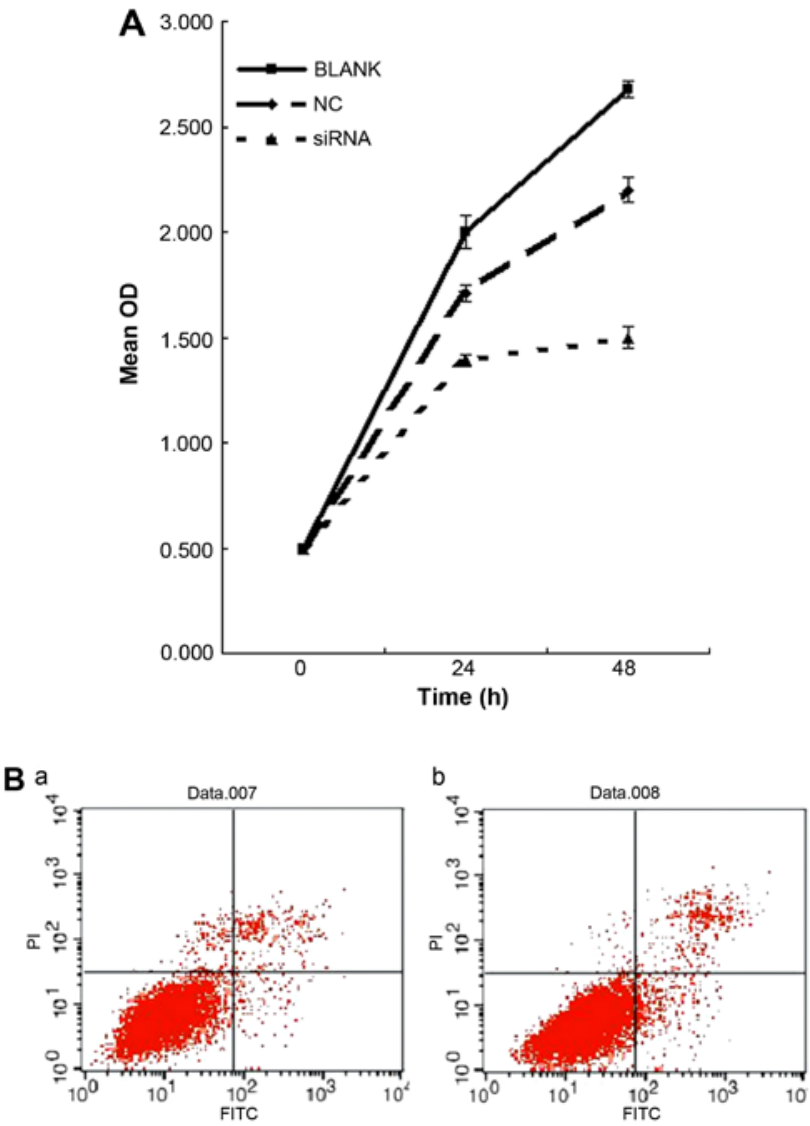

Figure 3. The overall level of cell proliferation was first tested by MTT staining. (A) Knockdown of NFATc1 decreased the number of proliferating cells by at least 2 -fold in the SKOV3 cells. (B) To explore the underlying mechanism by which NFATc1 induces ovarian tumor growth, we examined the effect of NFATc1 on cell apoptosis by flow cytometry. Knockdown of NFATc1 promoted cell apoptosis compared with the control cells (a, control; $\mathrm{b}$, siRNA). These results strongly suggest that NFATc1 promotes ovarian cancer development at least in part through regulation of cell proliferation and apoptosis.

subcutaneous tumor size in animals (Fig. 2B and Table V) were both distinctly reduced in cells treated with NFATc1 siRNA compared with the controls. These data showed that NFATc1 was upregulated in ovarian cancer cells and that silencing of NFATc1 diminished tumorigenicity and aggressiveness of the ovarian cancer cells both in vitro and in vivo.
Table V. Statistical analysis of the transplanted tumors in nude mice.

\begin{tabular}{lcccc}
\hline Groups & $\begin{array}{c}\text { Weight } \\
(\mathrm{g})\end{array}$ & $\begin{array}{c}\text { Volume } \\
\left(\mathrm{cm}^{3}\right)\end{array}$ & $\begin{array}{c}\text { Inhibition } \\
\text { rate }(\%)\end{array}$ & P-value \\
\hline A (Blank) & $2.03 \pm 0.35$ & $1.328 \pm 145$ & 0 & $\mathrm{P} 1$ \\
B (Control) & $1.98 \pm 0.78$ & $1.274 \pm 209$ & 0 & P2 \\
C (siRNA) & $0.87 \pm 0.32$ & $0.512 \pm 087$ & 57.08 & P3 \\
\hline
\end{tabular}

Comparison of each group with regard to weight and volume. P1, A:B $>0.05 ; \mathrm{P} 2, \mathrm{~B}: \mathrm{C}<0.05 ; \mathrm{P} 3, \mathrm{~A}: \mathrm{C}<0.05$.

Table VI. Inhibition rate of cell growth in each group.

\begin{tabular}{lrc}
\hline Group & \multicolumn{1}{c}{$24 \mathrm{~h}$} & $48 \mathrm{~h}$ \\
\hline A (Blank) & $9.0 \pm 0.50$ & $11.2 \pm 0.10$ \\
B (Control) & $9.5 \pm 1.60$ & $15.0 \pm 1.50$ \\
C (siRNA) & $47.3 \pm 0.78$ & $56.0 \pm 0.82$ \\
\hline
\end{tabular}

The inhibition group compared with each control group, $\mathrm{P}<0.05$.

Table VII. Apoptosis rate in each group.

\begin{tabular}{lccc}
\hline & Mean & SD & P-value \\
\hline NC & 3.7767 & 0.24028 & 0.000 \\
SiRNA & 6.9800 & 0.23000 &
\end{tabular}

Inhibition group (siRNA) compared with the control (NC) group, $\mathrm{P}=0.000$. SD, standard deviation.

Regulation of cell proliferation and cell apoptosis by NFATc1 siRNA in ovarian cancer cells. We first tested the overall level of cell proliferation by MTT staining. Knockdown of NFATc1 decreased the number of proliferating cells by at least 2 -fold in the SKOV3 cells (Fig. 3A; Table VI). To explore the underlying mechanism by which NFATc1 induces ovarian tumor growth, 

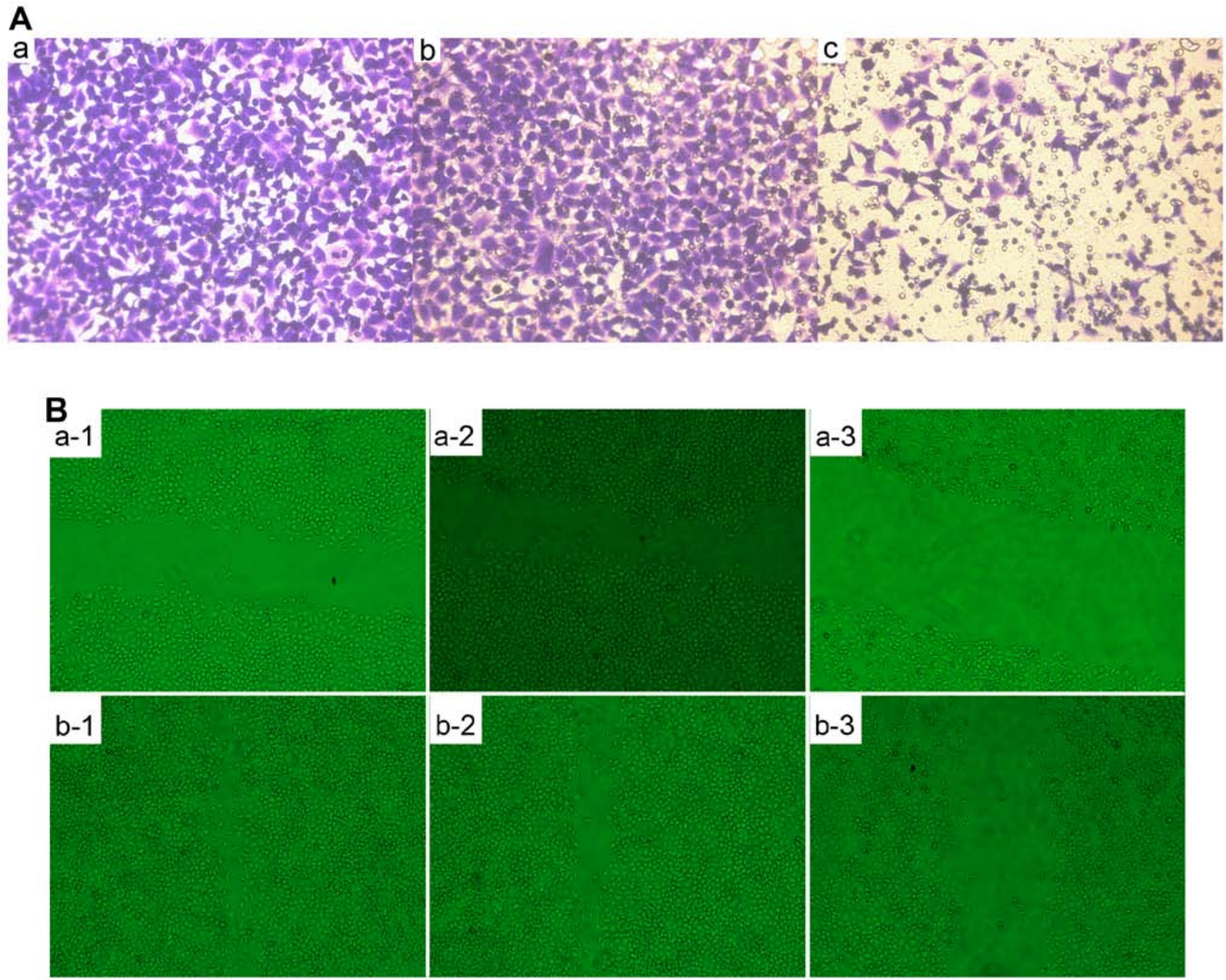

Figure 4. (A) The SKOV3 cells that migrated through the filter pores to the underside of the filter in (c) the experimental group were significantly increased compared with the cells in (a) the control and (b) the blank groups $(\mathrm{P}<0.05)$. (B) In the wound-healing assays, the distance migrated by cells after wounding a cell monolayer on plastic after treatment with NFATc1 siRNA and control was obtained. (B) The results are reported as migration indices, which represent the distance migrated by the control (a-1 and 2, b-1 and 2) or NFATc1 siRNA-treated (a-3, b-3) cells to close the wound.

Table VIII. Statistical analysis of SKOV3 cell invasion.

\begin{tabular}{lcc}
\hline Groups & No. of cells & P-value \\
\hline A (Blank) & $115 \pm 16$ & P1 \\
B (Control) & $111 \pm 18$ & P2 \\
C (siRNA) & $39 \pm 7$ & P3 \\
\hline
\end{tabular}

Comparison of each group of cells. $\mathrm{P} 1, \mathrm{~A}: \mathrm{B}>0.05 ; \mathrm{P} 2, \mathrm{~B}: \mathrm{C}<0.05 ; \mathrm{P} 3$, A:C $<0.05$.

we first examined the effect of NFATc1 on cell apoptosis by flow cytometry. As shown in Fig. 3B and Table VII, knockdown of NFATc1 promoted cell apoptosis compared with the control cells. These results strongly suggest that NFATc1 promotes ovarian development at least in part through regulation of cell proliferation and apoptosis.

Regulation of cell invasion and migration by NFATcl in ovarian cancer cells. The cells that migrated through the filter
Table IX. Statistical analysis of SKOV3 cell migration.

\begin{tabular}{llc}
\hline Groups & $24 \mathrm{~h}$ & $48 \mathrm{~h}$ \\
\hline A (Blank) & $42 \pm 9$ & $101 \pm 17$ \\
B (Control) & $40 \pm 10$ & $106 \pm 19$ \\
C (siRNA) & $15 \pm 4$ & $38 \pm 9$ \\
\hline
\end{tabular}

Comparison of each group of cells. P1, A:B $>0.05$; P2, B:C $<0.05$; $\mathrm{P} 3, \mathrm{~A}: \mathrm{C}<0.05$.

pores to the underside of the filter in the experimental group were significantly increased compared with the cells in the control and blank groups ( $\mathrm{P}<0.05$; Fig. $4 \mathrm{~A}$ and Table VIII). In the wound-healing assays, the distance migrated by the cells after wounding a cell monolayer on plastic after treatment with NFATc1 siRNA and control was obtained. The results (Fig. 4B, Table IX) are reported as migration indices, which represent the distance migrated by the control or NFATc1 siRNA-treated cells to close the wound, expressed relative 


\section{A}
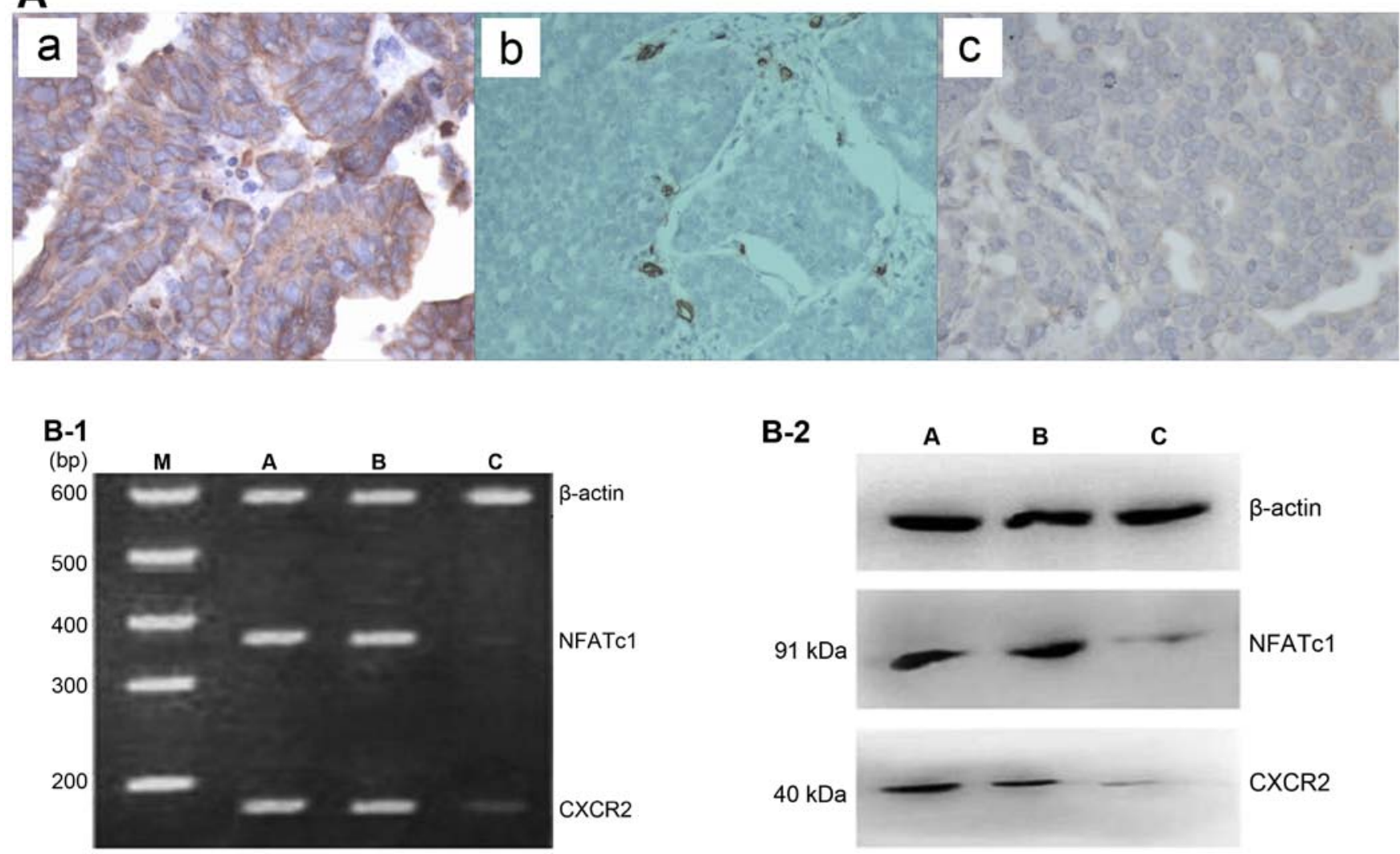

Figure 5. (A) Xenograft mouse tumor tissues generated from animals injected with SKOV3/NFATc1 siRNA-1169 were evaluated. The overall blood vessel density was decreased in tissues expressing NFATc1 siRNA, as indicated by the reduced number of tissue microvessels stained with CD34 (a, CK staining; $b$, control; c, siRNA). (B) We then detected the expression of CXCR2 in mouse tumor tissues treated with or without NFATc1 siRNA. We found that CXCR2 was significantly decreased in the xenograft tumor tissues treated with NFATc1 siRNA (lanes A and B, controls; lane C, siRNA). These results suggest that NFATc1 could promote ovarian cancer angiogenesis by activating CXCR2, which may be mediated through the upstream signaling of VEGFA.

Table X. Statistical analysis of MVD.

\begin{tabular}{lrc}
\hline Group & MVD & P-value \\
\hline A (Blank) & $12.00 \pm 1.65$ & P1 \\
B (Control) & $11.47 \pm 0.32$ & P2 \\
C (siRNA) & $5.36 \pm 0.34$ & P3 \\
\hline
\end{tabular}

Comparison of each group of genes. $\mathrm{P} 1, \mathrm{~A}: \mathrm{B}>0.05 ; \mathrm{P} 2, \mathrm{~B}: \mathrm{C}<0.05$; $\mathrm{P} 3, \mathrm{~A}: \mathrm{C}<0.05$.

to the distance migrated by the control-treated cells. In the SKOV3 cells, NFATc1 siRNA significantly decreased migration compared with the non-targeted controls $(\mathrm{P}<0.05)$. This result was consistent with results obtained for this cell line in the Transwell assay, as described above.

Regulation of ovarian cancer angiogenesis by NFATcl. It is well known that VEGFA (angiogenic factor A) promotes tumor angiogenesis. As previously mentioned, research has shown that VEGFA activates calcineurin and induces NFATc1 transcriptional activity. To confirm whether NFATc1 is associated with angiogenesis, we first examined the xenograft mouse tumor tissues generated from animals injected with SKOV3/ NFATc1 siRNA-1169. The overall blood vessel density was
Table XI. Statistical analysis of NFATc1 and CXCR2 mRNA.

\begin{tabular}{lccc}
\hline Group & NFATc1 & CXCR2 & P-value \\
\hline A (Blank) & $0.912 \pm 0.001$ & $0.925 \pm 0.005$ & P1 \\
B (Control) & $0.896 \pm 0.001$ & $0.882 \pm 0.003$ & P2 \\
C (siRNA) & $0.265 \pm 0.003$ & $0.338 \pm 0.001$ & P3 \\
\hline
\end{tabular}

Comparison of each group of genes. P1, A:B >0.05; P2, B:C <0.05; P3, A:C $<0.05$.

decreased in tissues expressing NFATc1 siRNA, as indicated by the reduced number of tissue microvessels stained with CD34 (Fig. 5A and Table X). In addition, we then detected the expression of CXCR2 in mouse tumor tissues treated with or without NFATc1 siRNA. We found that CXCR2 was significantly decreased in the xenograft tumor tissues treated with NFATc1 siRNA (Fig. 5B and Tables XI and XII). These results suggest that NFATc1 promotes ovarian cancer angiogenesis by activating CXCR2, which may be mediated through the upstream signaling of VEGFA.

Association of NFATcl with ovarian cancer patient survival. Immunohistochemical staining of NFATc1 in 93 specimens 
Table XII. Statistical analysis of NFATc1 and CXCR2 protein in the SKOV 3 cells.

\begin{tabular}{lccc}
\hline Group & NFATc1 & CXCR2 & P-value \\
\hline A (Blank) & $0.907 \pm 0.001$ & $0.926 \pm 0.005$ & P1 \\
B (Control) & $0.827 \pm 0.005$ & $0.913 \pm 0.003$ & P2 \\
C (siRNA) & $0.295 \pm 0.003$ & $0.382 \pm 0.001$ & P3 \\
\hline
\end{tabular}

Comparison of the same protein in each group. $\mathrm{P} 1, \mathrm{~A}: \mathrm{B}>0.05 ; \mathrm{P} 2$, $\mathrm{B}: \mathrm{C}<0.05 ; \mathrm{P} 3, \mathrm{~A}: \mathrm{C}<0.05$.

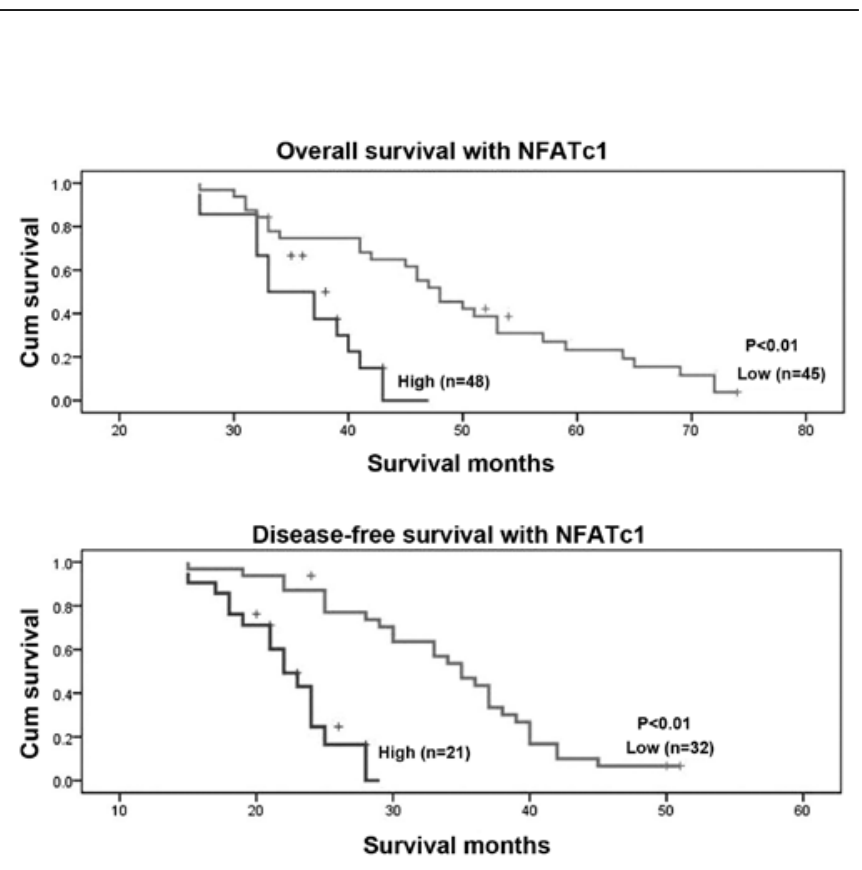

Figure 6. Immunohistochemical staining of NFATc1 in 93 specimens of high-grade ovarian carcinoma showed that both the cell membrane and cytoplasm of epithelial cancer tissues were specifically stained with NFATc1, whereas little NFATc1 expression was detected in benign ovarian tumors. (Representative images are shown in Fig. 1A). Statistically, patients with low NFATc1 expression lived longer (mean, 49 months) than patients with high NFATc1 expression (mean, 36 months; $\mathrm{P}<0.01$ ). In terms of disease-free survival, patients with high NFATc1 expression also relapsed earlier in the course of the disease (mean, 22 months) than did patients with low expression (mean, 35 months; $\mathrm{P}<0.01$ ).

of high-grade ovarian carcinoma showed that both the cell membrane and cytoplasm of epithelial cancer tissues were specifically stained with NFATc1, whereas little NFATc1 expression was detected in benign ovarian tumors. Representative images are shown in Fig. 1A. Statistically, patients with low NFATc1 expression lived longer (mean, 49 months) than patients with high NFATc1 expression (mean, 36 months; $P<0.01$, Fig. 6). In terms of disease-free survival, patients with high NFATc1 expression also relapsed earlier in the course of the disease (mean, 22 months) than did patients with low expression (mean, 35 months; $\mathrm{P}<0.01$; Fig. 6). Univariate and multivariate analyses of FIGO stage, age at diagnosis, and NFATc1 expression showed all of these factors to be independent prognostic factors for overall survival, whereas FIGO stage and age at diagnosis were independent prognostic factors for disease-free survival (Table XIII). These data suggest that NFATc1 overexpression is significantly associated with EOC prognosis.
Table XIII. Association of NFATc1 expression with patient characteristics.

\begin{tabular}{|c|c|c|c|c|}
\hline & $\begin{array}{c}\text { NFATc1 } \\
\text { low }\end{array}$ & $\begin{array}{l}\text { NFATc1 } \\
\text { high }\end{array}$ & Total & P-value \\
\hline \multicolumn{5}{|l|}{ Stage } \\
\hline I-II & 6 & 0 & 6 & \\
\hline III-IV & 39 & 48 & 87 & \\
\hline Total & 45 & 48 & 93 & 0.005 \\
\hline \multicolumn{5}{|l|}{ Family history } \\
\hline Yes & 31 & 14 & 45 & \\
\hline No & 25 & 20 & 45 & \\
\hline Unknown & 2 & 1 & 3 & \\
\hline Total & 58 & 35 & 93 & 0.03 \\
\hline \multicolumn{5}{|c|}{ Age at diagnosis (years) } \\
\hline$\geq 60$ & 30 & 21 & 51 & \\
\hline$<60$ & 28 & 14 & 42 & \\
\hline Total & 58 & 35 & 93 & 0.281 \\
\hline \multicolumn{5}{|l|}{ Tumor size (cm) } \\
\hline$\leq 2$ & 9 & 3 & 12 & \\
\hline$>2$ & 9 & 72 & 81 & \\
\hline Total & 18 & 75 & 93 & 0.041 \\
\hline \multicolumn{5}{|l|}{ Relapse } \\
\hline Yes & 27 & 15 & 42 & $0.045^{\mathrm{a}}$ \\
\hline Progressive disease & 19 & 18 & 37 & $0.005^{\mathrm{b}}$ \\
\hline No & 6 & 1 & 7 & $0.05^{\mathrm{c}}$ \\
\hline Unknown & 5 & 2 & 7 & \\
\hline Total & 57 & 36 & 93 & \\
\hline \multicolumn{5}{|l|}{ Ascites } \\
\hline No & 3 & 2 & 5 & $0.781^{\mathrm{d}}$ \\
\hline Yes & 36 & 27 & 63 & $0.24^{\mathrm{e}}$ \\
\hline Unknown & 18 & 5 & 23 & \\
\hline Minimal & 2 & 0 & 2 & $0.121^{\mathrm{f}}$ \\
\hline Total & 59 & 34 & 93 & \\
\hline
\end{tabular}

${ }^{a}$ Yes vs. progressive disease; ${ }^{b}$ progressive disease vs. no; ${ }^{c}$ yes vs. no. ${ }^{\mathrm{d}}$ no vs. yes; ${ }^{\mathrm{e}}$ yes vs. minimal; ${ }^{\mathrm{f}}$ no vs. minimal.

The association between patient clinical characteristics and NFATc1 expression is summarized in Table XIII. Low levels of NFATc1 expression were noted in a higher proportion of patients with early-stage carcinoma compared with patients who had late-stage disease with high levels of NFATc1 expression $(\mathrm{P}=0.005)$. A higher proportion of patients with a positive family history of cancer had low NFATc1 expression compared with patients with no family history of cancer $(\mathrm{P}=0.03)$. Patients who did not experience a relapse had low NFATc1 expression compared with patients whose disease progressed $(\mathrm{P}=0.005)$.

\section{Discussion}

EOC is an extremely aggressive disease, with most patients having metastases or extensive local invasion at the time of 
diagnosis (27). Little is known regarding the potential role of NFATc1 in the process of ovarian cancer cell growth, motility or dissemination. In the present study, we provide strong evidence that NFATc1 plays a critical role in ovarian cancer progression by regulating cell proliferation, apoptosis, invasion and migration.

As a transcription factor, NFATc1 has been extensively investigated in terms of lymphocyte activation in the immune system (28). However, increasing evidence has shown that NFATc1 is commonly expressed in mammalian tissues and plays important roles in various malignancies (29), but the function of the important nuclear factor in EOC has not been well defined. In the present study, we first characterized NFATc1 expression in benign and malignant ovarian cysts. We found that NFATc1 was expressed in 93 of the 96 EOC specimens and in only 6 of the 30 benign ovarian tumor specimens and 5 of the 30 benign ovarian endometriosis specimens. In addition, NFATc1 mRNA and protein expression were significantly increased in EOC $(\mathrm{P}<0.05)$. To explore the potential effect of NFATc1 in EOC, we silenced NFATc1 by green fluorescent small interfering RNA and infected SKOV3 cells using Lipofectamine ${ }^{\mathrm{TM}}$ 2000. Our data showed that NFATc1 RNA and protein were significantly decreased by siRNA in the SKOV3 cell line and in transplanted tumor tissues, leading to cancer cell growth arrest and an increase in apoptotic cells. Our finding that NFATc1 is overexpressed in a high proportion of EOC led us to investigate whether it also could contribute to the motility of ovarian cancer cells. Depletion of NFATc1 in SKOV3 cells caused a marked reduction in motility, observed in both Transwell and wound-healing experiments. In further experiments, we used animal models to investigate whether NFATc1 is important in ovarian cancer cell proliferation and dissemination, and we subsequently showed that a reduction in NFATc1 gene expression inhibited the tumor growth rate and tumor size and promoted ovarian cancer invasion and migration through strengthening tumor angiogenesis. It is well known that VEGFA promotes cancer tissue angiogenesis (30), and research has found that VEGFA activates calcineurin and induces NFATc1 transcriptional activity. To confirm whether NFATc1 is associated with angiogenesis, we first examined transplanted tumor tissues generated from animals injected with SKOV3/NFATc1 siRNA-1169. The overall blood vessel density was notably decreased in tissues expressing NFATc1 siRNA. In addition, we then detected the expression of CXCR2 (a critical angiogenic factor) downstream of the VEGFA signaling (31) in mouse tumor tissues treated with or without NFATc1 siRNA. We found that CXCR2 was significantly decreased in xenograft tumor tissues treated with NFATc1 siRNA. These results suggest that NFATc1 could promote ovarian cancer angiogenesis by activating CXCR2, which may be mediated through the upstream signaling of VEGFA. Finally, we showed that NFATc1 overexpression in ovarian cancer tissue was associated with poor survival and early relapse in high-grade serous ovarian cancer patients.

In summary, we provide the first study indicating that NFATc1 is overexpressed in both ovarian cancer cell lines and ovarian cancer tissues from patients but not in benign ovarian tumors, suggesting that NFATc1 may be a potential target for ovarian cancer treatment. Thus, NFATc1 antagonists that can effectively inhibit ovarian cancer cell growth may be a potential strategy for ovarian cancer treatment. Our observations that depletion of NFATc1 in ovarian cancer cell lines diminishes their motility suggest the possible involvement of the protein in the motility and consequently the dissemination of ovarian cancer cells. Since NFATc1 overexpression is also associated with angiogenesis in animals and poor survival among ovarian cancer patients, indicating that this transcription factor may also contribute to the aggressive nature of this cancer, NFATc1 may be a novel prognostic marker for ovarian cancer and a potential target for therapeutic intervention. Further studies into the role of NFATc1 in ovarian cancer are merited.

\section{Acknowledgements}

The present study was supported by the National Science Foundation of China (no. 81402126).

\section{References}

1. Shaw JP, Utz PJ, Durand DB, Toole JJ, Emmel EA and Crabtree GR: Identification of a putative regulator of early $\mathrm{T}$ cell activation genes. Science 241: 202-205, 1988

2. Pan MG, Xiong Y and Chen F: NFAT gene family in inflammation and cancer. Curr Mol Med 13: 543-554, 2013.

3. Jain J, McCaffrey PG, Valge-Archer VE and Rao A: Nuclear factor of activated T cells contains Fos and Jun. Nature 356: 801-804, 1992.

4. Wu Y, Borde M, Heissmeyer V, Feuerer M, Lapan AD, Stroud JC, Bates DL, Guo L, Han A, Ziegler SF, et al: FOXP3 controls regulatory $\mathrm{T}$ cell function through cooperation with NFAT. Cell 126: 375-387, 2006.

5. Robbs BK, Cruz AL, Werneck MB, Mognol GP and Viola JP: Dual roles for NFAT transcription factor genes as oncogenes and tumor suppressors. Mol Cell Biol 28: 7168-7181, 2008.

6. Flockhart RJ, Diffey BL, Farr PM, Lloyd J and Reynolds NJ: NFAT regulates induction of COX-2 and apoptosis of keratinocytes in response to ultraviolet radiation exposure. FASEB J 22: 4218-4227, 2008

7. Chen L, Glover JN, Hogan PG, Rao A and Harrison SC: Structure of the DNA-binding domains from NFAT, Fos and Jun bound specifically to DNA. Nature 392: 42-48, 1998.

8. Abdoli $\mathrm{R}$ and Najafian $\mathrm{N}$ : $\mathrm{T}$ helper cells fate mapping by Co-stimulatory molecules and its functions in allograft rejection and tolerance. Int J Organ Transplant Med 5: 97-110, 2014.

9. Medyouf H, Alcalde H, Berthier C, Guillemin MC, dos Santos NR, Janin A, Decaudin D, de Thé H and Ghysdael J: Targeting calcineurin activation as a therapeutic strategy for T-cell acute lymphoblastic leukemia. Nat Med 13: 736-741, 2007.

10. Macián F, López-Rodríguez C and Rao A: Partners in transcription : NFAT and AP-1. Oncogene 20: 2476-2489, 2001.

11. Stevenson AS, Gomez MF, Hill-Eubanks DC and Nelson MT: NFAT4 movement in native smooth muscle. A role for differential Ca ${ }^{2+}$ signalling. J Biol Chem 276: 15018-15024, 2001.

12. Flockhart RJ, Armstrong JL, Reynolds NJ and Lovat PE: NFAT signalling is a novel target of oncogenic BRAF in metastatic melanoma. Br J Cancer 101: 1448-1455, 2009.

13. Tripathi P, Wang Y, Coussens M, Manda KR, Casey AM, Lin C, Poyo E, Pfeifer JD, Basappa N, Bates CM, et al: Activation of NFAT signaling establishes a tumorigenic microenvironment through cell autonomous and non-cell autonomous mechanisms. Oncogene 33: 1840-1849, 2014.

14. Baumgart S, Chen NM, Siveke JT, König A, Zhang JS, Singh SK, Wolf E, Bartkuhn M, Esposito I, Heßmann E, et al: Inflammation-induced NFATc1-STAT3 transcription complex promotes pancreatic cancer initiation by KrasG12D. Cancer Discov 4: 688-701, 2014.

15. Kawahara T, Kashiwagi E, Ide H, Li Y, Zheng Y, Miyamoto Y, Netto GJ, Ishiguro H and Miyamoto H: Cyclosporine A and tacrolimus inhibit bladder cancer growth through down-regulation of NFATc1. Oncotarget 6: 1582-1593, 2015. 
16. Pennisi A, Ling W, Li X, Khan S, Shaughnessy JD Jr, Barlogie B and Yaccoby S: The ephrinB2/EphB4 axis is dysregulated in osteoprogenitors from myeloma patients and its activation affects myeloma bone disease and tumor growth. Blood 114: 1803-1812, 2009.

17. Schabbauer G, Schweighofer B, Mechtcheriakova D, Lucerna M, Binder BR and Hofer E: Nuclear factor of activated T cells and early growth response-1 cooperate to mediate tissue factor gene induction by vascular endothelial growth factor in endothelial cells. Thromb Haemost 97: 988-997, 2007.

18. Kavitha CV, Deep G, Gangar SC, Jain AK, Agarwal C and Agarwal R: Silibinin inhibits prostate cancer cells- and RANKL-induced osteoclastogenesis by targeting NFATc1, NF- $\kappa$ B and AP-1 activation in RAW264.7 cells. Mol Carcinog 53: 169-180, 2014.

19. Murray OT, Wong CC, Vrankova K and Rigas B: Phospho-sulindac inhibits pancreatic cancer growth: NFATc1 as a drug resistance candidate. Int J Oncol 44: 521-529, 2014.

20. Yang G, Rosen DG, Liu G, Yang F, Guo X, Xiao X, Xue F, Mercado-Uribe I, Huang J, Lin SH, et al: CXCR 2 promotes ovarian cancer growth through dysregulated cell cycle, diminished apoptosis, and enhanced angiogenesis. Clin Cancer Res 16: 3875-3886, 2010.

21. Matsuo Y, Raimondo M, Woodward TA, Wallace MB, Gill KR, Tong Z, Burdick MD, Yang Z, Strieter RM, Hoffman RM, et al: CXC-chemokine/CXCR2 biological axis promotes angiogenesis in vitro and in vivo in pancreatic cancer. Int J Cancer 125: 1027-1037, 2009.

22. Wu B, Wang Y, Lui W, Langworthy M, Tompkins KL, Hatzopoulos AK, Baldwin HS and Zhou B: Nfatcl coordinates valve endocardial cell lineage development required for heart valve formation. Circ Res 109: 183-192, 2011.

23. Lee JH, Bhang DH, Beede A, Huang TL, Stripp BR, Bloch $\mathrm{KD}$, Wagers AJ, Tseng YH, Ryeom S and Kim CF: Lung stem cell differentiation in mice directed by endothelial cells via a BMP4-NFATc1-thrombospondin-1 axis. Cell 156: 440-455, 2014.
24. Jang GH, Park IS, Yang JH, Bischoff J and Lee YM: Differential function of genes regulated by VEGF-NFATc1 signaling pathway in migration of pulmonary valve endothelial cells. FEBS Lett 584: 141-146, 2010

25. Stankunas K, Ma GK, Kuhnert FJ, Kuo CJ and Chang CP: VEGF signaling has distinct spatiotemporal roles during heart valve development. Dev Biol 347: 325-336, 2010.

26. Pickens SR, Chamberlain ND, Volin MV, Gonzalez M, Pope RM, Mandelin AM II, Kolls JK and Shahrara S: Anti-CXCL5 therapy ameliorates IL-17-induced arthritis by decreasing joint vascularization. Angiogenesis 14: 443-455, 2011.

27. Bachmann C, Krämer B, Brucker SY, Stäbler A, Fend F, Wallwiener D, Grischke EM and Rothmund R: Relevance of pelvic and para-aortic node metastases in early-stage ovarian cancer. Anticancer Res 34: 6735-6738, 2014.

28. Shin B, Yu J, Park ES, Choi S, Yu J, Hwang JM, Yun H, Chung YH, Hong KS, Choi JS, et al: Secretion of a truncated osteopetrosis-associated transmembrane protein 1 (OSTM1) mutant inhibits osteoclastogenesis through down-regulation of the B lymphocyte-induced maturation protein 1 (BLIMP1)-nuclear factor of activated T cells c1 (NFATc1) axis. J Biol Chem 289: 35868-35881, 2014.

29. Kawahara T, Kashiwagi E, Ide H, Li Y, Zheng Y, Ishiguro H and Miyamoto H: The role of NFATc1 in prostate cancer progression: Cyclosporine A and tacrolimus inhibit cell proliferation, migration, and invasion. Prostate 75: 573-584, 2015.

30. Guérin F, Wagner M, Liné A, Zappa M, Fasseu M, Paradis V, Vilgrain V, Van Beers BE, Legagneux J, Moreau R, et al: Hepatic proliferation and angiogenesis markers are increased after portal deprivation in rats: A study of molecular, histological and radiological changes. PLoS One 10: e0125493, 2015.

31. Adinolfi E, Raffaghello L, Giuliani AL, Cavazzini L, Capece M, Chiozzi P, Bianchi G, Kroemer G, Pistoia V and Di Virgilio F: Expression of P2X7 receptor increases in vivo tumor growth. Cancer Res 72: 2957-2969, 2012. 